PSICOLOGÍA

IBEROAMERICANA
Psicología Iberoamericana ISSN: 1405-0943

revista.psicologia@ibero.mx

Universidad Iberoamericana, Ciudad de México México

\title{
Evaluación de usabilidad, deseo de jugar y sentido de presencia en ambientes virtuales para el tratamiento del juego compulsivo
}

González-Perellón, Jaime M.; Durón-Figueroa, Raúl; Bouchard, Stéphane; Cárdenas-López, Georgina Evaluación de usabilidad, deseo de jugar y sentido de presencia en ambientes virtuales para el tratamiento del juego compulsivo

Psicología Iberoamericana, vol. 29, núm. 1, 2021

Universidad Iberoamericana, Ciudad de México, México

Disponible en: https://www.redalyc.org/articulo.oa?id=133967526003

DOI: https://doi.org/10.48102/pi.v29i1.302

\section{(c) (1)}

Esta obra está bajo una Licencia Creative Commons Atribución 4.0 Internacional. 
Psicología Iberoamericana, vol. 29, núm. 1,2021

Universidad Iberoamericana, Ciudad de México, México

Recepción: 04 Noviembre 2020 Aprobación: 12 Marzo 2021

DOI: https://doi.org/10.48102/ pi.v29i1.302

Redalyc: https://www.redalyc.org/ articulo.oa?id=133967526003

Financiamiento

Fuente: Consejo Nacional de Ciencia y

Tecnología (CONACYT)

$\mathrm{N}^{\circ}$ de contrato: 262546

Beneficiario: Jaime Miguel González

Perellón
Artículos

\section{Evaluación de usabilidad, deseo de jugar y sentido de presencia en ambientes virtuales para el tratamiento del juego compulsivo}

Usability, Gambling Desire, and Presence evaluation of Virtual Environments for the Treatment of Compulsive

Gambling

Jaime M. González-Perellón jaime.miguel.gonzalez.p@gmail.com Universidad Nacional Autónoma de México (UNAM), México https://orcid.org/0000-0001-9075-8082 Raúl Durón-Figueroa raulduron7@gmail.com Universidad Nacional Autónoma de México (UNAM), México

D https://orcid.org/0000-0002-5520-6706

Stéphane Bouchard Stephane.Bouchard@uqo.ca Université du Québec en Outaouais, Canadá

https://orcid.org/0000-0002-5995-340X

Georgina Cárdenas-López cardenas.georgina@gmail.com Universidad Nacional Autónoma de México (UNAM), México

https://orcid.org/0000-0003-4569-1944

Resumen: El DSM-5 clasifica el juego compulsivo como un trastorno caracterizado por la necesidad de jugar. La efectividad de la terapia cognitivo-conductual (TCC) se destaca en el tratamiento del juego compulsivo. Sin embargo, el uso de técnicas tradicionales de TCC ha mostrado algunas limitaciones. La Realidad Virtual (RV) es una técnica de exposición que ha mostrado ventajas sobre el control de estímulos y un mejor acceso a pensamientos disfuncionales. En el presente estudio se usó un diseño longitudinal de medidas repetidas, 30 participantes conformados por 14 mujeres y 16 hombres, de entre 18 y 65 años con una pantalla DSM-5 para problemas de juego (NODS) $>1$ evaluaron dos escenarios de realidad virtual. El estudio se llevó a cabo en la Ciudad de México. Los resultados en deseos de jugar, el Slater-Usoh-Steed (SUS) y otras medidas de seguimiento, muestran que la exposición a los ambientes virtuales produce un malestar físico leve, es asociada con sensaciones de presencia y que los efectos de la exposición disminuyen con el tiempo. Estos hallazgos sugieren que los ambientes de realidad virtual son viables para su uso como técnica de exposición.

Palabras clave: juego patológico, realidad virtual, trastornos adictivos, trastornos no relacionados a sustancias, TCC.

Abstract: The DSM-5 classifies compulsive gambling as a non-substance-related addiction disorder characterized by the need to gamble. The effectiveness of cognitivebehavioural therapy (CBT) stands out in the treatment of pathological gambling. However, the use of CBT traditional techniques to manage the problem has shown some limitations. Virtual Reality (VR) is an exposure technique that has shown advantages over stimulus control and better access to dysfunctional thoughts. The present study used a one-way repeated measures design. The sample included 30 
participants, comprised of 14 women and 16 men, between the ages of 18 and 65 years old. Data collection included a DSM-5 screen for problem gambling (NODS) $>1$. Participants evaluated two virtual reality scenarios. The results, which used Slater-UsohSteed (SUS), and other follow-up measures, show that virtual environments generate mild physical sickness, are associated with presence sensations and that the desire to gamble is associated with exposure decreases over time. These findings suggest that virtual reality environments are viable for use as an exposure technique.

Keywords: gambling, virtual reality, addictive disorders, non-substance-related disorders, CBT.

\section{Introducción}

El juego compulsivo se ha definido como la falta de control de impulsos y deseos excesivos a participar en juegos de azar y, a su vez, una conducta descontrolada relacionada con las apuestas, mismo que fue reconocido como un trastorno mental en la tercera versión del Diagnostic and Statistical Manual of Mental Disorders (American Psychiatric Association, 1980), en el cual era clasificado dentro de los trastornos de control de los impulsos. Más recientemente, en la $5^{a}$ edición del Diagnostic and Statistical Manual of Mental Disorders (American Psychiatric Association, 2013), dicho trastorno ha sido clasificado dentro de los trastornos adictivos y no relacionados con sustancias, donde es definido como un comportamiento de juego disfuncional recurrente y persistente que genera una interferencia significativa en las actividades personales, familiares y laborales. Por su parte, la clasificación del International Statistical Classification of Diseases and Related Health Problems (World Health Organization, 2019) lo ha definido como un patrón de comportamiento de juego persistente y recurrente, siendo presencial o en línea (internet), en el cual el individuo no tiene control, dando prioridad sobre otras actividades y continúa jugando a pesar de las consecuencias negativas.

En cuanto a la prevalencia de dicho trastorno, se han realizado diversos estudios a nivel mundial. De acuerdo con el DSM-5 (American Psychiatric Association, 2013), se ha encontrado una prevalencia de .2\% a. $3 \%$ en población general. Asimismo, la prevalencia a lo largo de la vida en mujeres es de $.2 \%$ y en hombres de .6\%. En una revisión sistemática (Calado \& Griffiths, 2016), donde se incluyeron 69 estudios enfocados en medir la prevalencia, se encontraron prevalencias anuales que van del.1\% a $5 \%$ de personas con problemas de juego, siendo Estados Unidos y Hong Kong las más altas (5\%), y Suiza (.1\%) y España (.2\%) las más bajas. En cuanto a los criterios de Juego Patológico se encontraron prevalencias que van del .1 \% al $2.2 \%$, siendo Hong Kong (2.2\%) y Macao (1.8\%) las más altas; los países con índices más bajos fueron Alemania, Gran Bretaña y Noruega con .2\% y Dinamarca con .1\%.

En México existen pocos estudios en cuanto a la epidemiología de dicho trastorno. Un estudio reciente (Villatoro et al., 2018) encontró que el $24.5 \%$ de población entre 12 y 65 años de edad ha jugado, por lo menos una vez, algún juego de azar en su vida, donde el .3\% de la población cumple los criterios de juego compulsivo, y en adolescentes 
el $.4 \%$ presenta los criterios. Asimismo, se encontró que los hombres presentan prevalencias estadísticamente significativas más altas. Por otro lado, en un estudio realizado en población universitaria $(n=2183)$, se encontró una prevalencia de $12.1 \%$ para jugadores problema y $7.4 \%$ para jugadores patológicos (Martínez, 2014).

Considerando las diversas problemáticas psicosociales que se derivan del juego compulsivo, en los últimos años se ha prestado especial atención en modelos de intervención eficaces para el manejo de dicho trastorno. En general, se han logrado identificar distintos programas de tratamiento que han demostrado su eficacia, donde destaca la terapia cognitivo conductual (TCC), terapia motivacional, terapia cognitiva y terapia farmacológica, por mencionar las principales (Potenza et al., 2019). A pesar de que los distintos modelos mencionados han mostrado resultados clínicos positivos, la TCC ha demostrado ser la más eficaz y más utilizada en el tratamiento de juego patológico (Menchon et al., 2018).

Dentro de los programas basados en TCC se logran identificar distintos componentes basados en teorías cognitivas y conductuales. Las técnicas cognitivas, como lo es la reestructuración cognitiva y prevención de recaídas, están enfocadas en modificar los sesgos o distorsiones cognitivas asociadas al juego compulsivo. Por su parte, las técnicas conductuales, predominando la desensibilización, control de estímulos y técnicas de exposición con prevención de respuesta, están centradas en la modificación conductual asociada al juego mediante estrategias para limitar el uso de dinero, estrategias de evasión a situaciones de riesgo y estrategias de autocontrol mediante la exposición a estímulos que provocan el deseo de jugar (Echeburúa et al., 2014). A pesar de que la TCC ha demostrado su eficacia en el tratamiento del juego patológico, también ha mostrado algunas limitaciones importantes a considerar. En el caso de la exposición por imaginación, se ha encontrado que pueden presentarse algunas limitaciones importantes en cuanto a la capacidad de imaginar situaciones relacionadas al juego que provoquen ansiedad o deseos de jugar, resultando en una falla en el momento de generalizar en situaciones de la vida real. Por otro lado, en cuanto a la exposición in vivo, se han identificado problemas relacionados con la falta de control en situaciones de la vida real, ya que el terapeuta no tiene la capacidad de controlar los estímulos asociados como moderar el momento y tiempo de la exposición (Bouchard et al., 2017; Chrétien et al., 2018). Considerando dichas limitaciones, se ha encontrado que una alternativa prometedora en las técnicas de exposición ha sido el uso de la realidad virtual (RV), misma que permite tener un mayor control de estímulos, permitiendo un mayor acceso a pensamientos disfuncionales asociados al juego y estímulos asociados que inducen el deseo de jugar en los individuos; lo cual resulta útil, por ejemplo, en la exposición con prevención de respuesta (Chrétien et al., 2018; Giroux et al., 2013; Park et al., 2015).

Aunque la RV ha resultado ser una alternativa prometedora en el tratamiento del juego patológico, existen pocos ambientes virtuales validados. Loranger et al. (2011), mediante el Laboratorio de Ciberpsicología de la Universidad de Quebec, desarrollaron y validaron 
dos ambientes virtuales para el tratamiento del juego patológico. Dichos ambientes fueron diseñados para simular situaciones relacionadas al juego con apuestas, mismos que se dividen en un bar y un casino virtual que incluyen video-loterías y máquinas de apuestas, además de otras situaciones que simulan comportamientos característicos de los jugadores patológicos. En cuanto a la validación, se encontró que se presentó un mayor deseo de jugar, activación fisiológica, y estado de ánimo positivo en los jugadores frecuentes que en no jugadores, tanto en máquinas reales como en los juegos incluidos en los ambientes virtuales. A su vez, dichos resultados reflejaron que los ambientes virtuales evocaron el mismo deseo de jugar que las máquinas de apuestas reales, aún sin estar utilizando dinero real, lo cual permite utilizar dichos ambientes en contextos terapéuticos (Bouchard et al., 2014). Posteriormente, utilizando los ambientes virtuales mencionados, Bouchard et al. (2017) realizaron tres estudios enfocados en: 1) evaluar el deseo de jugar mediante la exposición a los ambientes virtuales en comparación con juegos de apuestas reales y un grupo control con un juego sin apuestas, 2) evaluar el uso de un protocolo breve de TCC con el uso de RV, y 3) evaluar el uso de un protocolo con el uso de RV y determinar la seguridad del uso de dichos ambientes en cuanto al deseo de jugar posterior al tratamiento. Los resultados mostraron que la inmersión en la $\mathrm{RV}$ provoca el deseo de jugar, siendo mayor el deseo en aquellas personas que juegan más frecuentemente que las personas que no suelen jugar y, a su vez, se encontró que la RV es de gran utilidad en la TCC, donde se logran identificar mayores situaciones de riesgo y pensamientos disfuncionales en los jugadores patológicos, que el inducir el deseo de jugar dentro de los ejercicios de prevención de recaídas tiene una relación significativa con los resultados del tratamiento, y que dichos ambientes resultan seguros en cuanto a los malestares físicos que pudiera provocar la exposición en los escenarios virtuales. Por último, en los estudios mencionados se mostró que el uso de RV no se asocia con mayores deseos de jugar persistentes posteriores a la exposición, en comparación con otros tipos de exposición, lo cual brinda seguridad y control en el tratamiento mediante RV.

Considerando la problemática que representa el juego patológico en cuanto al grado de interferencia en la vida diaria de las personas que lo padecen, así como la falta de atención especializada en población mexicana, resulta importante contar con más investigación sobre el tema para lograr formular tratamientos eficaces basados en evidencia. El presente estudio, por medio del Laboratorio de Enseñanza Virtual y Ciberpsicología, de la Facultad de Psicología de la U.N.A.M., en conjunto con el Laboratorio de Ciberpsicología de la Universidad de Quebec, tuvo por objetivo evaluar el malestar físico, el deseo de jugar, así como el sentido de presencia generado por los ambientes virtuales desarrollados por el grupo de Canadá. Se plantea que la exposición a los ambientes virtuales puede ser asociada con variaciones en deseos de jugar y sensaciones de presencia, que el malestar físico producido por los ambientes puede ser bien tolerado y que los efectos de la exposición cambiarán con el tiempo. También se pretende que, mediante el presente trabajo sobre 
las respuestas a la exposición y usabilidad, se pueda profundizar en el conocimiento sobre las respuestas a los ambientes empleados y abordar la viabilidad de la exposición con RV. Indagar sobre algunas de las condicionantes y sobre la evaluación de los usuarios a la exposición con $\mathrm{RV}$, para integrar la técnica en futuros estudios y de esa manera evaluar la eficacia del tratamiento mediante RV con el uso de los escenarios virtuales en cuestión.

\section{Método}

Se utilizó un diseño longitudinal de medidas repetidas de una vía, intrasujetos (Wallace \& Green, 2002) considerando el tiempo después de la exposición como factor de variación en las respuestas de los participantes, con valores observados post-exposición, $0.5,1,12$ y 24 horas posteriores al uso de los ambientes. El método corresponde a la evaluación de usabilidad de los escenarios. Se buscó pilotear un conjunto de criterios sobre las respuestas a la RV. Se abordaron conforme a los supuestos de Stewart y Jefferson (2007) aspectos relacionados con el estudio de las conductas de juego. Se empleó como método no estadístico de eliminación para controlar efectos sobre las variables criterio, excluir los casos que pudieran formar parte de una muestra clínica de jugadores patológicos y los participantes con puntaciones de susceptibilidad al juego nula.

\section{Planteamiento del problema}

Es necesario el conocimiento de las variaciones en respuesta a los ambientes de juego en RV para abordar su uso como estrategia de exposición en el tratamiento del juego compulsivo.

Hipótesis: La exposición a los ambientes virtuales se relaciona con la expresión de mayores deseos de jugar que disminuyen con el paso del tiempo. Hay diferencias intra-sujetos en las medidas del deseo de jugar que son determinadas por el factor del tiempo desde la exposición. Hay un sentido de presencia dentro de los ambientes evaluados, las puntuaciones de presencia están por arriba del punto medio entre los usuarios. Los ambientes de RV son bien tolerados, las puntuaciones de malestar son moderadas entre los usuarios.

\section{Participantes}

Un total de 33 participantes fueron reclutados a través de volantes, panfletos y carteles que se distribuyeron en el campus de la Universidad Nacional Autónoma de México en la Ciudad de México y por Internet para este estudio. Se consideraron 30 participantes para la inclusión en la muestra por sus respuestas en una entrevista y los criterios mínimos del DSM-5 para jugador problemático, episódico, en remisión inicial, sin nivel de gravedad conforme a sus medidas en NODS $>1$, los participantes debían calificar en el cuestionario con un valor mayor a 1 en 
los criterios de susceptibilidad al juego. Los participantes que calificaron con más de 4 criterios clínicos para jugador patológico se excluyeron del estudio. Se obtuvo información sobre algunas características individuales como sexo, edad, escolaridad y ocupación como se muestra en la Tabla 1. EL rango de edad en los participantes fue de entre 18 y 65 años. Fue obtenido el consentimiento informado antes de cada sesión y las respuestas de los participantes fueron anónimas y confidenciales. Los participantes seleccionados fueron asignados aleatoriamente a las sesiones de exposición.

\section{Tabla 1}

\section{Tabla 1}

Demografía y características clínicas

\begin{tabular}{llrr}
\hline & Puntuaciones mayores a uno en NODS & $(\mathrm{n}=30)$ & \\
\cline { 2 - 5 } & & $\mathrm{N}$ & $\%$ \\
\hline \multirow{2}{*}{ Sexo } & Mujeres & 14 & 46.7 \\
& Hombres & 16 & 53.3 \\
& & & \\
& Estudiante & 23 & 76.7 \\
& Pasante & 6 & 20 \\
Escolaridad & Profesionista & 1 & 3.3 \\
& & & \\
& Bachillerato & 28 & 93.3 \\
& Universidad & 2 & 6.7 \\
\hline Edad & & Media & DE \\
NODS & & 23.2 & 4.3 \\
\hline
\end{tabular}

Nota. $N=30$. Todos los participantes eran mayores de 18 años, las puntuaciones en NODS son siempre mayores a $1(n=30$, NODS > 1). DE = Desviación Estándar.

\section{Medidas}

Se convocó un panel de expertos especialistas en TCC a los que se les facilitó la versión usada para cada instrumento del estudio. Se les solicitó revisar y calificar los instrumentos para evaluar la representatividad, comprensión, ambigüedad y claridad de cada ítem. Se excluyeron los ítems que no reflejaran consistencia entre las calificaciones de los evaluadores. 
Se hizo uso de una entrevista no estructurada basada en los criterios para jugador problemático del DSM-5 y un seguimiento sobre la susceptibilidad al trastorno. Antes de la exposición se tomaron medidas sociodemográficas. Finalizando se evaluaron los resultados de la exposición, sobre el deseo de participar en juegos de apuestas, y sobre otras dimensiones que se esperaría pudieran presentar variaciones debidas a la exposición como autocontrol, presencia en al ambiente virtual, y sobre malestar físico y efectos secundarios. Se realizó la evaluación utilizando los siguientes instrumentos.

\section{Susceptibilidad al trastorno de juego patológico}

Se midió a través de la escala NORC DSM-IV Screen for Gambling Problems (NODS) (Gerstein et al., 1999), Adaptación de Becoña (2004). Consistió de 17 ítems en un cuestionario de dos partes que incluyen preguntas sobre los eventos de vida y sobre el último año. Punto de corte $>1$. El análisis psicométrico original del NODS fue implementado por sus autores en una muestra de 40 individuos en programas de tratamiento por problemas de juego. Las propiedades psicométricas del instrumento se han evaluado con muestras internacionales incluyendo 12,521 participantes en línea, de 105 países, con una proporción de alrededor de $3 \%$ para población de México y Latinoamérica (Williams $\&$ Volberg, 2010). Se consideran 4 o más de los criterios contenidos en el DSM-4 para el diagnóstico clínico de jugador patológico. Para este estudio se realizó una prueba de consistencia interna mediante un análisis de confiabilidad alfa de Cronbach, se realizó también un análisis de componentes principales con rotación varimax. Las correlaciones interítem rondaron entre 0.22 hasta 0.79 . La solución final fue consistente con los resultados de Petry et al. (2013), para la solución con 4-9 criterios del DSM-IV el instrumento se comporta como una escala unidimensional. En la solución final un eigenvalue mayor de 1 con 3.44 representó un factor que explica el $49 \%$ de la varianza. Las cargas factoriales para la mayoría de los criterios rondan entre 0.62 y 0.77 , y las comunalidades se encuentran entre 0.39 y 0.60 . La prueba de esfericidad de Barlett's fue significativa (279.6, $\mathrm{gl}=21, \mathrm{p}<0.001)$, y el indicador de adecuación del tamaño de la muestra Kaiser-Meyer-Olkin fue adecuado (.833). El alfa del instrumento total fue de .84 .

\section{Deseo de apostar, autocontrol y malestar}

MiTratamiento (MiT) (Bouchard et al., 2016), escala para evaluar resultados de exposición a escenarios virtuales de juego que consistió en 11 ítems que conforman distintas dimensiones para evaluar excitación, emoción, deseo de jugar, autocontrol, creencias, oportunidad de jugar, personas y situación estímulo y efectos secundarios. Los reactivos se califican como puntos porcentuales asignados por los participantes de $0 \mathrm{a}$ 100, y como promedios de puntuaciones que van del 0 al 9 . Se convocó a 
9 expertos en el área de tratamiento cognitivo conductual que evaluaron cada ítem con base en características de representatividad, comprensión, ambigüedad y claridad. Para conservar los reactivos se determinó el grado de acuerdo entre expertos con el coeficiente kappa de Cohen $(\kappa>.45)$. Se obtuvo una concordancia $\kappa>.80$ por parejas de expertos para cada criterio de la escala. El instrumento se aplicó como un cuestionario de autoinforme al terminar la exposición, a los 30 minutos, 60 minutos, 12 horas y 24 horas después de la exposición.

\section{Sentido de presencia}

Para evaluar el sentido de presencia en el ambiente virtual se usó un cuestionario basado en Slater-Usoh-Steed (SUS) (Slater et al., 1994). Para medir la presencia, cinco ítems fueron calificados en una escala de siete puntos que miden el sentido de estar allí, el grado en que el escenario de RV se vuelve más real que la realidad y la "localidad", es decir, el grado en que el escenario de RV es pensado como un lugar visitado.

\section{Equipo}

El equipo de realidad virtual consistió en una computadora CPU Intel Original 2160 con $1.80 \mathrm{GHz}$ y $1 \mathrm{~GB}$ de RAM, sistema operativo Windows XP Professional y una pantalla montada en la cabeza Vusix iWear AV920 con auriculares incluidos para estímulos de audio. Los participantes interactuaron con el VE utilizando un mouse o las teclas de flecha del teclado. Los ambientes virtuales (Loranger et al., 2011) consisten en tres ambientes virtuales diseñados para llevar a cabo sesiones de exposición mediante realidad virtual (Figura 1). El primer ambiente, consiste en un escenario neutral dentro de un departamento que tiene por objetivo llevar a cabo una navegación libre y que el usuario pueda familiarizarse con el uso de los controles, así como detectar posibles malestares generados por la exposición virtual. Los dos escenarios referentes a las situaciones relacionadas con juegos de apuestas consisten, por un lado, en un bar en el cual el usuario tiene la posibilidad de interactuar en la barra de bebidas, con una máquina expendedora de lotería, y jugar en una máquina tragamonedas. Por otro lado, se presenta un casino donde el usuario puede interactuar en el lugar y jugar en las distintas máquinas de apuestas que se visualizan. 
Figura 1

Ambientes Virtuales para exposición en juego patológico

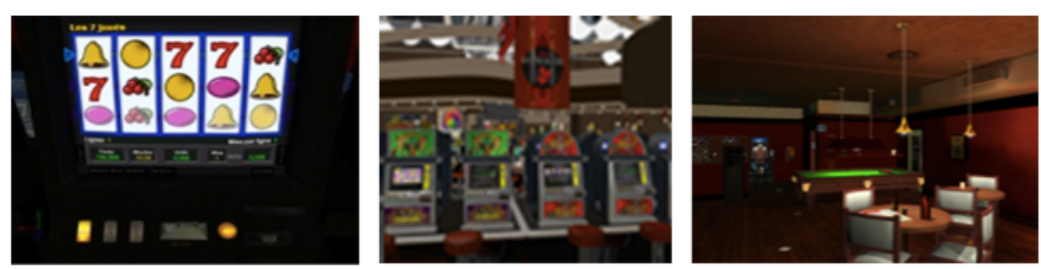

Nota. Imágenes de los ambientes virtuales de exposición. Las máquinas, el piso de casino y los escenarios de bares fueron diseñados para facilitar la identificación de situaciones en juego de apuestas.

\section{Figura 1}

\section{Procedimiento}

Inicialmente, los participantes interesados fueron contactados vía telefónica o por correo electrónico y se asignó una cita para participar en el estudio. Los participantes fueron asignados aleatoriamente y de forma individual a cada una de las sesiones de entrevista y exposición en las instalaciones de la universidad. Una vez que se les explicó en qué consistía el protocolo, se expresó la confidencialidad en el tratamiento de los datos de sus respuestas y la necesidad de su consentimiento informado para comenzar. Para los participantes entrevistados que cumplieron los criterios para la exposición, el investigador explicó el protocolo de aplicación e informó a los participantes sobre los posibles riesgos y malestares que se pueden presentar durante la exposición a los ambientes virtuales (cybersickness), como dolor de cabeza o náuseas. Antes de realizar la exposición se colectó la firma del aval del consentimiento informado, se aplicó la primera parte de los cuestionarios relacionada con características sociodemográficas. Antes de la inmersión se preparó el ambiente virtual conforme al manual de los desarrolladores, y se le explicó al participante cómo se ejecutaría la sesión y cuáles serían los objetivos. Se guio al participante en la inmersión en realidad virtual, solicitando familiarizarse primero con un escenario neutral para, posteriormente, realizar la navegación en los dos ambientes de juegos con apuestas. Durante la inmersión, se solicitó verbalizar las situaciones con las que tenía contacto. Después del recorrido del circuito de los ambientes o el equivalente en un período de aproximadamente 20 minutos, el participante pudo terminar la exposición y dejar el escenario virtual. Después de la inmersión se verificó si el participante tenía algún tipo de malestar, se utilizó la sección de efectos secundarios contenido en el cuestionario de exposición y se aplicaron las entrevistas y los instrumentos para evaluar el deseo de jugar y el sentido de presencia que generaron los ambientes virtuales. Una vez terminada la aplicación, se explicó a los participantes que se tendrían que realizar medidas de seguimiento una hora, 12 horas y 24 horas después de la exposición, y se establecieron los 
horarios correspondientes para realizar llamadas telefónicas y recopilar los datos en cuestión.

\section{Análisis estadistico}

Para el análisis de los datos se utilizó el software SPSS versión 22. Para examinar el efecto de la exposición en el deseo de jugar, se enviaron las puntuaciones de los participantes en las medidas de reporte a una serie de ANOVAs 1 (medida: deseo de jugar, autocontrol) x 5 (tiempo: base, $30 \mathrm{~min}, 60 \mathrm{~min}, 12 \mathrm{hr}, 24 \mathrm{hr}$ ) con medidas repetidas para el factor tiempo. Se obtuvieron medidas de tendencia central y desviación para las puntuaciones de las variables de sentido de presencia y malestar. Se realizaron correlaciones para explorar la relación entre la susceptibilidad al juego patológico, el deseo de jugar y el autocontrol después de la exposición.

\section{Resultados}

La Figura 2 presenta la media del deseo de jugar y la media de autocontrol después de la exposición a los ambientes de bar y casino. Las puntuaciones de los participantes relacionadas con el deseo de jugar son más elevadas después de entrar en los ambientes de bar y casino virtuales, y decaen en las evaluaciones posteriores. Los participantes expresan sentir un mayor deseo de jugar inmediatamente después de la exposición. Sobre el efecto de exposición a los ambientes virtuales, al someter las puntuaciones a un ANOVA de un factor de medidas repetidas. El test de esfericidad de Mauchly indica que el supuesto de esfericidad no se cumple para el efecto del tiempo en el deseo de participar en juegos de apuestas $\left(\chi^{2}(9)=31, \mathrm{p}<0.05\right)$; por lo tanto, los grados de libertad se han corregido con la estimación de esfericidad de Greenhouse-Geisser $(\varepsilon=0.65)$. Se encontraron diferencias estadísticamente significativas, $\mathrm{F}(2.6,75.4)=10.8, \mathrm{p}<.05, \eta 2 \mathrm{p}=0.27$ que indican la caída del efecto de la exposición en el deseo de jugar con el tiempo. Por otro lado, las medidas de autocontrol sobre el deseo de jugar muestran una tendencia diferente. Las puntuaciones más bajas de autocontrol se presentan inmediatamente después de la exposición a los ambientes virtuales y aumentan en las evaluaciones posteriores. En el caso de las medidas de autocontrol en el ANOVA de un factor de medidas repetidas. El test de esfericidad de Mauchly indica que el supuesto de esfericidad no se cumple para el efecto del tiempo en el autocontrol $\left(\chi^{2}(9)=45.4\right.$, $\mathrm{p}<0.05)$; por lo tanto los grados de libertad se han corregido con la estimación de esfericidad de Huyn-Feldt $(\varepsilon=0.433)$. No pudieron observarse diferencias estadísticamente significativas con puntuaciones de autocontrol frente al factor del tiempo de exposición, $\mathrm{F}(2.7,78.6)=1.824$, $\mathrm{p}=.155$.

El puntaje promedio para presencia en el SUS fue $21.4(D E=6.4)$, es decir con una calificación promedio por arriba del punto medio de 
la escala. Estos resultados sugieren que los participantes experimentaron presencia en los ambientes virtuales (Tabla 2).

La puntuación promedio en la escala de exposición para malestar y efectos secundarios fue de $26(D E=32.4)$, los efectos secundarios después de la exposición a los ambientes de bar y casino cuando llegan a presentarse se encuentran en niveles relativamente moderados.

\section{Figura 2}

\section{Medidas del deseo de jugar y de autocontrol seguidas de la exposición}

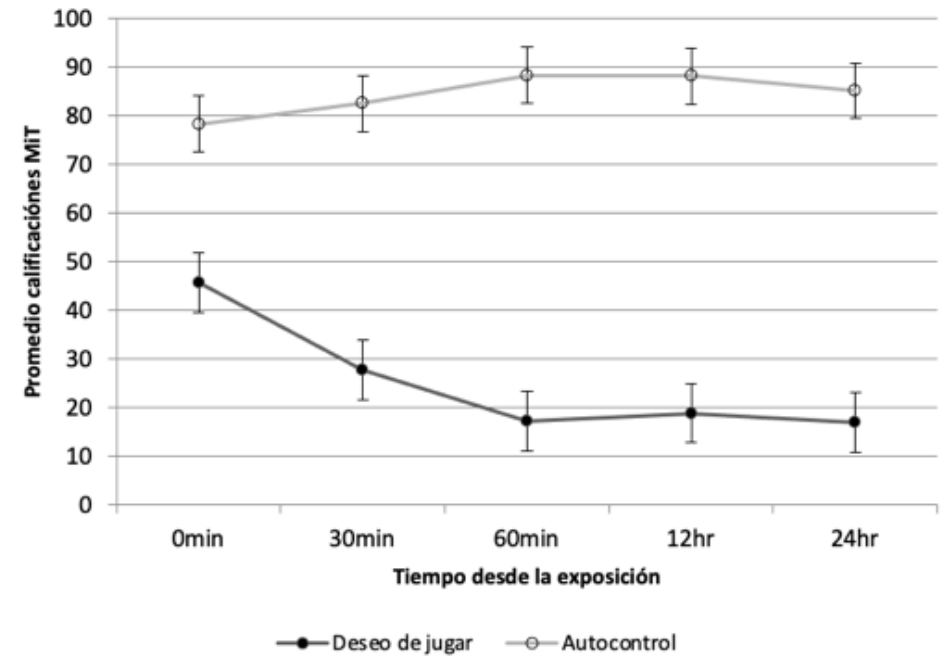

Nota. Promedio de calificaciones del cuestionario MiT relacionadas con el deseo de jugar ( $n=30$; círculos negros) y promedio de calificaciones del cuestionario MiT relacionadas con el autocontrol sobre el deseo de jugar ( $n=30$; círculos blancos). Error estándar de la media en la serie de puntos y líneas. Las etiquetas para el eje de las $\mathrm{x}$ denotan medidas tomadas después de la exposición a los ambientes de casino.

\section{Figura 2}

Es posible suponer que no hay una clara correspondencia de las variables clínicas con las medidas de exposición, después de que se encontraron resultados mixtos entre la calificación del NODS y las puntuaciones de las medidas evaluadas referentes a la exposición. No se encontraron relaciones estadísticamente significativas entre las puntuaciones del NODS con la excitación, la emoción por apostar o el deseo de jugar después de la exposición. Para la correspondencia de las calificaciones del NODS y las medidas de autocontrol, solamente al seleccionar los casos con las puntuaciones más altas en el NODS (NODS $>3$ ) se encontró una correspondencia en las puntuaciones relacionadas con las medidas tomadas después de la exposición, $\mathrm{r}=-.991, \mathrm{p}=000$. Pudo observarse que las calificaciones en el NODS estaban relacionadas significativamente con las medidas de atribución o creencias sobre la 
suerte (¿cree que la probabilidad de ganar en los juegos de azar es debido únicamente a la suerte?), $\mathrm{r}=-.414, \mathrm{p}=.023$.

Tabla 2

Tabla 2

Evaluación de la experiencia en realidad virtual

\begin{tabular}{|c|c|c|c|c|c|}
\hline \multicolumn{6}{|c|}{ Cuestionario exposición a RV } \\
\hline & Media(DE) & Media(DE) & Media(DE) & Media(DE) & Media(DE) \\
\hline \multicolumn{6}{|l|}{ Tiempo después } \\
\hline de la exposición & $-\min$ & $30 \mathrm{~min}$ & $60 \mathrm{~min}$ & $12 \mathrm{hr}$ & $24 \mathrm{hr}$ \\
\hline Malestar & $26(32.4)$ & - & - & - & - \\
\hline Excitación & $31(33.6)$ & - & - & - & - \\
\hline Emoción & $34.2(29.4)$ & - & - & - & - \\
\hline Deseo de jugar & $45.7(33.5)$ & $27.7(30.4)$ & $17.2(24.7)$ & $18.8(25.4)$ & $16.9(29)$ \\
\hline Autocontrol & $78.3(27)$ & $82.5(27.2)$ & $88.3(19.8)$ & $88.1(25.9)$ & $85.1(31.5)$ \\
\hline Creencias (suerte) & $43.3(31)$ & $39.8(30.5)$ & $41.5(30)$ & $42.1(30.1)$ & $41.6(31.1)$ \\
\hline \multicolumn{6}{|l|}{ Oportunidad de } \\
\hline jugar & - & $.63(1.5)$ & $.57(1.48)$ & $.57(1.1)$ & $.67(1.3)$ \\
\hline \multicolumn{6}{|l|}{ Exposición } \\
\hline \multicolumn{6}{|l|}{ (personas y } \\
\hline situación estímulo) & $.50(1.3)$ & $.63(1.3)$ & $.43(.73)$ & $.70(1.2)$ & $.63(1.6)$ \\
\hline Sus & $21.4(6.4)$ & - & - & - & - \\
\hline
\end{tabular}

Cuando fueron seleccionados los casos con puntuaciones en el NODS más altas esta relación dejó de ser significativa, $\mathrm{r}=-.449, \mathrm{p}=.193$. También se encontró una correspondencia entre las calificaciones en la prueba y las medidas relacionadas con oportunidad de jugar, $\mathrm{r}=.558, \mathrm{p}$ $=.001$, y presencia de personas o situaciones estímulo contenidas en el bar y el casino virtuales, $\mathrm{r}=.407, \mathrm{p}=.026$.

\section{Discusión}

El principal objetivo del estudio fue evaluar el deseo de jugar asociado con la exposición a los ambientes virtuales, desarrollados por el grupo de investigación del Laboratorio de Ciberpsicología de la Universidad de Quebec (2011). La exposición a los ambientes virtuales se relaciona con la expresión de mayores deseos de jugar que disminuyen con el paso del tiempo. Los resultados de Loranger et al. (2011) y Bouchard et al. (2017), indican que la exposición a los escenarios de RV genera 
el deseo de participar en juegos de apuestas. Los resultados del presente estudio, podrían ser tomados en cuenta para considerar que la exposición a los escenarios de RV está relacionada con el deseo de participar en juegos de apuestas en situaciones de exposición distintas en contexto a la intervención original. Al comparar nuestros resultados con los resultados obtenidos en el estudio original de Loranger et al. (2011) y de Bouchard et al. (2017), se confirmó que los ambientes virtuales de bar y casino se relacionan con un mayor deseo de participar en juegos de apuestas. Además, las reacciones psicológicas mientras se juega con las máquinas de lotería, y los tragamonedas del ambiente virtual, podrían permitir detectar quienes están en mayor riesgo de involucrarse en episodios de juego patológico. Tanto el ambiente del dispensador de lotería virtual, como el casino de RV podrían resultar interesantes para su uso como parte de un programa de tratamiento de mayor alcance que incluya la prevención de recaídas.

En este estudio, el deseo por jugar fue mayor después de la exposición a los escenarios, y disminuyó conforme al paso del tiempo. Además de las medidas relacionadas con el deseo de participar en juegos de apuestas, también se evaluó la usabilidad del escenario en respuestas para distintas dimensiones, considerando la sensación de presencia, malestar, y la correspondencia con variables clínicas de control usadas en el NODS. Pudo observarse una correspondencia entre la exposición al escenario y sensaciones de presencia. En general el escenario fue bien tolerado sin que se presentara un malestar considerable u otros efectos secundarios. Los resultados sobre la correspondencia entre las medidas relacionadas con la exposición y las variables clínicas, no reflejaron una relación muy consistente. La relación entre las respuestas del NODS no reveló que, en general, las diferencias en puntuaciones en la escala supusieran variaciones potenciales en los resultados relacionados con la exposición al escenario. Sobre estos alcances de los resultados en la teoría de la conducta de juego, podría ser sugerida una analogía con los hallazgos de Steenbergh et al. (2004), y Zack y Poulos (2005); los mensajes informacionales pueden tener impacto en las creencias irracionales del jugador y su respuestas frente al juego, y distintos aspectos cognoscitivos implícitos y de rasgo podrían estar involucrados en las intenciones que condicionan la intensidad de la conducta del jugador.

Entre las limitaciones para este estudio se encuentra el tamaño de la muestra y los referentes con población mexicana. Los alcances para una adecuada generalización en ciertos aspectos de los resultados se ve restringida por el número de participantes. Los criterios de validez psicométrica empleados para las medidas de susceptibilidad al juego patológico por ejemplo, deben ser considerados con cautela partiendo de un alcance condicionado. En general la validación de instrumentos para el juego compulsivo hechos exprofeso para población mexicana es muy escasa, y la disponibilidad de medidas es un problema importante. Cabe mencionar que pese a que el uso de la tecnología de RV ha existido por más de 20 años (Slater, 2009), la integración de este componente tecnológico para la práctica clínica no ha sido tan vertiginosa como su implementación 
en otras áreas. El argumento de que la forma en que se experimenta este tipo de ambientes pueda llevar a la expresión realista de conductas sigue siendo explorado, integrando cada vez más especialistas de distintas áreas para su estudio.

\section{Conclusión}

Pese a las limitaciones relacionadas con el tamaño de la muestra, así como la falta de consistencia entre los criterios clínicos estudiados para la presente investigación, referentes a las puntuaciones en el NODS entre los participantes y la variación en las respuestas frente a la exposición a los ambientes de RV, pensamos que este tipo de ambientes pueden ser considerados como una estrategia viable como técnica de exposición que se relaciona con el deseo de participar en juegos de apuestas. Las puntuaciones en el NODS no tienen una relación clara con el deseo de participar en juegos de apuestas, o con el autocontrol del deseo de participar en juegos de apuestas después de la exposición, pero la respuesta psicológica a los ambientes, y el acceso a pensamientos sobre el interés por apostar, expresado por los participantes en los registros de seguimiento podrían ser útiles para detectar el riesgo de participar en juegos de apuestas entre los usuarios.

El conocimiento sobre los alcances de la estrategia de exposición a ambientes de Realidad Virtual para el tratamiento del juego patológico con población mexicana, todavía es muy limitado. Sin embargo, los resultados preliminares parecen alentadores. Nuevas investigaciones podrían abarcar también contrastes en las medidas sobre el deseo de jugar y la inhibición, al comparar en condiciones controladas el efecto de exposición al ambiente virtual frente al uso de aplicaciones dispensadoras de lotería, juego por internet, mesas de apuestas y máquinas de casino reales. Podría ser evaluado el deseo de apostar mediante la exposición a los ambientes virtuales en comparación con juegos de apuestas reales y con grupos control de juego sin apuestas. La investigación futura sobre este tema podría enriquecerse en el marco de un programa de intervención que considerase la integración de los ambientes de Realidad Virtual frente a otras estrategias de exposición para el tratamiento del juego patológico y las recaídas.

\section{Agradecimientos}

El trabajo recibió el apoyo del Consejo Nacional de Ciencia y Tecnología (CONACYT) beca (No. 262546) para realizar el estudio doctoral y a solicitud del Candidato a Doctor Jaime Miguel González Perellón.

\section{Referencias}

American Psychiatric Association. (1980). Diagnostic and statistical manual of mental disorders ( $3^{\mathrm{a}}$ ed.). American Psychiatric Publishing. 
American Psychiatric Association. (2013). Diagnostic and statistical manual of mental disorders ( $5^{\mathrm{a}}$ ed.). American Psychiatric Publishing. https://doi.or g/10.1176/appi.books.9780890425596

Becoña, E. (2004). The prevalence of pathological gambling in Galicia according to the nods. Reduction in prevalence or better assessment of the disorder? Adicciones, 16(3), 173-184. https://doi.org/10.20882/adicciones.399

Bouchard, S., Giroux, I., \& Sévigny, S. (2016). Utiliser la réalité virtuelle pour augmenter l'efficacité du traitement du jeu pathologique. [Montréal, Québec]\#: Fonds de recherche du Québec - Société et culture. http://www.frqsc.gouv.qc.ca/documents/11326/2469199/PT_ BouchardS_annexes_realite-virtuelle.

Bouchard, S., Loranger, C., Giroux, I., Jacques, C., \& Robillard, G. (2014). Using virtual reality to provide a naturalistic setting for the treatment of pathological gambling. En C. Sík Lányi (Ed.), The thousand faces of virtual reality (pp. 3-21). https://doi.org/10.5772/59240

Bouchard, S., Robillard, G., Giroux, I., Jacques, C., Loranger, C., St-Pierre, M., Chrétien, M., \& Goulet, A. (2017). Using virtual reality in the treatment of gambling disorder: The development of a new tool for cognitive behavior therapy. Frontiers in Psychiatry, 8, 24. https://doi.org/10.3389/ fpsyt.2017.00027

Calado, F., \& Griffiths, M. D. (2016). Problem gambling worldwide: An update and systematic review of empirical research (2000-2015). Journal of Behavioral Addictions, 5(4), 592-613. https://doi.org/10.1556/2006. 5.2016 .073

Chrétien, M., Giroux, I., Goulet, A., Jacques, C., \& Bouchard, S. (2018). Using virtual reality to elicit dysfunctional thoughts in individuals with gambling disorder. Journal of Gambling Issues, 2018(38), 169-189. https://doi.org /10.4309/jgi.2018.38.9

Echeburúa, E., Salaberría, K., \& Cruz-Sáez, M. (2014). Nuevos retos en el tratamiento del juego patológico. Terapia Psicologica, 32(1), 31-40. https ://doi.org/10.4067/S0718-48082014000100003

Gerstein, D., Murphy, S., Toce, M., Hoffmann, J., Palmer, A., Johnson, R., Larison, C., Chuchro, L., Buie, T., Engelman, L., Volberg, R., Harwood, A., Tucker, A., Christiansen, E., Cummings, W., \& Sinclair, S. (1999). Gambling impact and behavior study: Report to the national gambling impact study commission. National Opinion Research Center at the University of Chicago. http://cloud9.norc.uchicago.edu/dlib/ngis.ht $\mathrm{m}$.

Giroux, I., Faucher-Gravel, A., St-Hilaire, A., Boudreault, C., Jacques, C., \& Bouchard, S. (2013). Gambling exposure in virtual reality and modification of urge to gamble. Cyberpsychology, Behavior, and Social Networking, 16(3), 224-231. https://doi.org/10.1089/cyber.2012.1573

Loranger, C., Bouchard, S., Boulanger, J., \& Robillard, G. (2011). Validation of two virtual environments for the prevention and treatment of pathological gambling. Journal of CyberTherapy and Rebabilitation, 4(2), 233-236.

Martínez, R. (2014). Prevalencia de juego patológico en estudiantes universitarios [Tesis de Maestría, Universidad Autónoma de Nuevo León]. Repositorio Académico Digital UANL. http://eprints.uanl.mx/id/eprint/7798. 
Menchon, J. M., Mestre-Bach, G., Steward, T., Fernández-Aranda, F., \& Jiménez-Murcia, S. (2018). An overview of gambling disorder: From treatment approaches to risk factors. F1000Research, 7. https://doi.org/1 0.12688/f1000research.12784.1

Park, C.-B., Park, S. M., Gwak, A. R., Sohn, B. K., Lee, J.-Y., Jung, H. Y., Choi, S.-W., Kim, D. J., \& Choi, J.-S. (2015). The effect of repeated exposure to virtual gambling cues on the urge to gamble. Addictive Behaviors, 41, 6164. https://doi.org/10.1016/j.addbeh.2014.09.027

Petry, N. M., Blanco, C., Stinchfield, R., Volberg, R., \& Research, G. (2013). An empirical evaluation of proposed changes for gambling diagnosis in the DSM-5 NIH Public Access. Addiction, 108(3), 575-581. https://doi.org /10.1111/j.1360-0443.2012.04087.x

Potenza, M. N., Balodis, I. M., Derevensky, J., Grant, J. E., Petry, N. M., VerdejoGarcia, A., \& Yip, S. W. (2019). Gambling disorder. Nature Reviews Disease Primers, 5(1). https://doi.org/10.1038/s41572-019-0099-7

Slater, M. (2009). Place illusion and plausibility can lead to realistic behaviour in immersive virtual environments. Biological Sciences, 364(1535), 35493557. https://doi.org/10.1098/rstb.2009.0138

Slater, M., Usoh, M., \& Steed, A. (1994). Depth of presence in virtual environments. Presence: Teleoperators and Virtual Environments, 3(2), 130-144. https://doi.org/10.1162/pres.1994.3.2.130

Steenbergh, T. A., Whelan, J. P., Meyers, A. W., May, R. K., \& Floyd, K. (2004). Impact of warning and brief intervention messages on knowledge of gambling risk, irrational beliefs and behaviour. International Gambling Studies, 4(1), 3-16. https://doi.org/10.1080/1445979042000224377

Stewart, S. H., \& Jefferson, S. (2007). Experimental methodologies in gambling studies. En G. Smith, D. Hodgins \& R. J. Williams (Eds.), Research and measurement issues in gambling studies (pp. 88-107). Elsevier Academic Press.

Villatoro, J. A., Resendiz, E., Bustos, M. N., Mujica, A. R., Medina-Mora, M. E., Cañas, V., Soto, I. S., Fleiz, C., \& Romero, M. (2018). Magnitude and extent of gambling disorder in the Mexican population. Salud Mental, 41(4), 157-167. https://doi.org/10.17711/SM.0185-3325.2018.024

Wallace, D., \& Green, S. B. (2002). Analysis of repeated measures designs with linear mixed models. En D. S. Moskowitz \& S. L. Hershberger (Eds.), Multivariate applications book series. Modeling intraindividual variability with repeated measures data: Methods and applications (pp. 103-134). Lawrence Erlbaum Associates Publishers.

Williams, C. J., \& Volberg, R. J. (2010). Best practices in the population assessment of problem gambling. Report prepared for the Ontario Problem Gambling Research Centre. https://opus.uleth.ca/handle/10133/1259

World Health Organization. (2019). International statistical classification of diseases and related health problems ( $11^{\mathrm{a}} \mathrm{ed}$.). WHO. https://icd.who.int/

Zack, M., \& Poulos, C. X. (2005). Implicit cognition in problem gambling. En Handbook of implicit cognition and addiction (pp. 379-392). Sage Publications. https://doi.org/10.4135/9781412976237.n24 
Jaime M. González-Perellón, et al. Evaluación de usabilidad, deseo de jugar y sentido de presencia en ambientes virtuales para el tratamient...

\section{Declaración de intereses}

Esta investigación se ha realizado dentro del proyecto S.613 de la Fundación Río Arronte y LEVyC. No tenemos ningún conflicto de intereses conocido que reportar. 